\title{
Comitê de Ética em Pesquisa da Escola de Enfermagem da USP: experiência dos primeiros seis anos*
}

\author{
RESEARCH ETHICS COMMITTEE OF THE UNIVERSITY OF SÃO PAULO NURSING \\ SCHOOL: EXPERIENCE OF THE FIRST SIX YEARS \\ COMITÉ DE ÉTICA EN INVESTIGACIÓN DE LA ESCUELA DE ENFERMERÍA DE LA \\ USP: EXPERIENCIA DE LOS PRIMEROS SEIS AÑOS DE FUNCIONAMIENTO
}

\author{
Maria Cristina Komatsu Braga Massarollo ${ }^{1}$, Paulina Kurcgant ${ }^{2}$, Maria de Fátima Prado Fernandes ${ }^{3}$
}

\begin{abstract}
RESUMO
O objetivo do estudo foi conhecer a experiência do Comitê de Ética em Pesquisa da Escola de Enfermagem da USP (CEP-EEUSP), nos seus primeiros seis anos. $O$ estudo exploratório, descritivo, retrospectivo e de abordagem quantitativa foi realizado pelo levantamento dos registros do CEP-EEUSP e dos 401 protocolos de pesquisa submetidos à apreciação, no período de 31 de agosto de 1998 a 17 de setembro de 2004 . Os resultados evidenciaram que $98,7 \%$ dos projetos submetidos eram de pesquisadores da EEUSP; $31,4 \%$ eram de dissertações de mestrado e $29,1 \%$ de iniciação científica. Quanto ao método, 43,8\% propunham a utilização do método qualitativo. Além disso, $99,2 \%$ dos projetos pertenciam à área temática do Grupo III; 58,8\% previam a utilização de instituições públicas e 31,7\% tinham profissionais, principalmente enfermeiros, como sujeitos das pesquisas. 0 Termo de Consentimento Livre e Esclarecido foi responsável por $56,4 \%$ das pendências. A experiência do CEP-EEUSP mostra sua progressiva consolidação.
\end{abstract}

\section{DESCRITORES}

Comitês de Ética em Pesquisa.

Ética em pesquisa.

Enfermagem.

\begin{abstract}
The aim of this study was to know the experience of the first six years of the Research Ethics Committee (REC) of the University of São Paulo Nursing School activities. The exploratory, descriptive, retrospective and quantitative study analised the records of the REC and of all the 401 research protocols submitted for appreciation from 31st August, 1998 to 17th September, 2004. The results showed that $98.7 \%$ of protocols were from in-house researchers; $31.4 \%$ were master degree dissertations and $29.1 \%$ were graduate students scientific initiation. Qualitative methods were used by $43.8 \%$. Furthemore, $99.2 \%$ were classified as Group III thematic area; $58.8 \%$ researches were conducted in public institutions and $31.7 \%$ enrolled professionals, mostly nurses as research subjects. Informed Consent accounts for $56.4 \%$ of the REC's querries. The experience of the REC of the University of São Paulo Nursing School shows its progressive consolidation.
\end{abstract}

\begin{abstract}
RESUMEN
El objetivo de este estudio fue conocer la experiencia del Comité de Ética en Investigación de la Escuela de Enfermería de la USP en los primeros seis años de funcionamiento. Se trata de un estudio exploratorio, descriptivo, retrospectivo e de abordaje cuantitativo realizado con base en los registros del CEP-EEUSP y de los 401 protocolos de investigación presentados para evaluación en el período del 31 de agosto de 1998 hasta 17 de septiembre de 2004. Los resultados mostraron que $98,7 \%$ de los proyectos fueron presentados por investigadores de la EEUSP; 31,4\% eran de trabajos de maestría y $29,1 \%$ de iniciación científica. Cuanto a los métodos utilizados, $43,8 \%$ de los proyectos eran relativos al abordaje cualitativo. Además, 99,2\% pertenecían a la área temática del Grupo III; 58,8\% propusieron la utilización de instituciones públicas y $31,7 \%$ tenían profesionales, principalmente enfermeros, como sujetos investigados. El Termo de Consentimiento Libre e Informado fue el responsable por $56,4 \%$ de las pendencias. La experiencia del CEP-EEUSP muestra su progresiva consolidación.
\end{abstract}

\section{DESCRIPTORES}

Comités de Ética en Investigación.

Ética en investigación.

Enfermería.

\author{
KEY WORDS \\ Ethics Committees, Research. \\ Nursing.
}




\section{INTRODUÇÃO}

Historicamente, os Comitês de Ética em Pesquisa (CEPs) nascem como resposta às implicações morais do desenvolvimento técnico-científico na área biomédica, depois que foi desvendado que é possível, em nome da pesquisa e do progresso do conhecimento, cometer crimes hediondos contra a humanidade e contra os mais elementares direitos do cidadão(1).

A necessidade de criação dos comitês de ética, para apreciação dos protocolos de pesquisa, já era apontada na Declaração de Helsinque ${ }^{(2)}$, nas Diretrizes Éticas Internacionais para a Pesquisa Biomédica em Seres Humanos $(\mathrm{ClOMS})^{(3)}$ e na Resolução CNS 01/88 $8^{(4)}$. Com a Resolução CNS 196/96 $6^{(5)}$ foi regulamentada, no Brasil, a criação dos CEPs, que prevê a necessidade de todo projeto de pesquisa envolvendo seres humanos ser apreciado por um CEP antes de sua realização.

O CEP deve ser constituído por colegiado com número não inferior a sete membros, incluindo a participação de profissionais da área da saúde, das ciências exatas, sociais e humanas e, pelo menos, um membro da sociedade representando os usuários. Deve ter a participação de pessoas dos dois sexos, não podendo ter mais que a metade de seus membros pertencentes à mesma categoria profissional. A composição de cada CEP deve ser definida a critério da instituição, sendo pelo menos metade dos membros com experiência em pesquisa ${ }^{(5)}$. É importante que os pesquisadores incorporem a importância da ética na pesquisa, explicitando as questões éticas em seus projetos. Os abusos e deslizes éticos não serão inteiramente evitados por diretrizes ou documentos. $O$ efetivo controle ético envolve, principalmente, a sensibilização dos pesquisadores relativa à ética na pesquisa. A exigência de uma conduta ética pode indignar as pessoas, por parecer que é evidente demais e, portanto, desnecessária a colocação dessa questão. Entretanto, o fato é que as considerações de natureza ética nem sempre recebem a atenção apropriada ${ }^{(6)}$. Assim, os CEPs colocam um outro olhar nos projetos de pesquisa, fazendo com que os pesquisadores, e patrocinadores quando existem, não sejam os únicos a apreciarem se os seus projetos estão em conformidade com as diretrizes estabelecidas. As resistências, quando existem, originam-se, em especial, daqueles que acreditam que a competência ética sempre acompanha a competência técnica ${ }^{(7)}$. Cabe ressaltar que a apresentação dos projetos ao CEP não visa constituir obstáculos para a realização da pesquisa, mas, ao contrário, estimulá-las sob a égide da ética e da proteção do ser humano(8).

Frente à vivência do CEP-EEUSP, decidiu-se pela realização deste estudo, cuja finalidade é o resgate das atividades desenvolvidas na fase inicial do Comitê, que possi- bilita não só compor a história do CEP-EEUSP, como, também, visualizar a sua evolução.

\section{OBJETIVOS}

Geral: Conhecer a experiência do Comitê de Ética em Pesquisa da Escola de Enfermagem da Universidade de São Paulo, desde sua constituição, em agosto de 1998, até setembro de 2004.

Específicos: Descrever a composição e as atividades do CEP-EEUSP; apresentar o número de projetos avaliados pelo CEP-EEUSP; conhecer a procedência dos pesquisadores; verificar os métodos mais utilizados para o desenvolvimento das pesquisas; identificar o propósito acadêmico do desenvolvimento desses trabalhos; conhecer quais os sujeitos alvos dos estudos e a natureza das instituições onde as pesquisas seriam realizadas; verificar o número de projetos financiados e a origem do financiamento; identificar projetos de áreas temáticas especiais; relatar o resultado da apreciação dos projetos pelo CEP-EEUSP e identificar as causas mais incidentes de pendência.

\section{MÉTODO}

Foi feito um estudo exploratório, descritivo, retrospectivo e de abordagem quantitativa. Após autorização da instituição e concordância do CEP-EEUSP para utilização de seus documentos, foi realizado um levantamento dos seus registros, do período de 31 de agosto de 1998 a 17 de setembro de 2004, incluindo os referentes aos 401 protocolos de pesquisa submetidos à apreciação, no período em questão.

Dos 401 projetos registrados no período, todos foram utilizados para análise da atividade anual do Comitê (número de projetos submetidos por ano) e da procedência dos pesquisadores. Como houve a solicitação da retirada de dois projetos pelos pesquisadores, antes da apreciação, foram analisados efetivamente 399 projetos.

Neste trabalho, a caracterização dos membros do Comitê, tanto titulares como suplentes, que totalizaram 18 membros, incluiu categoria profissional, sexo e maior titulação acadêmica.

Os dados foram coletados com a utilização de um instrumento contendo os itens necessários para o alcance dos objetivos e submetidos à análise estatística descritiva, utilizando o programa SPSS for Windows versão 9.0 (Statistical Package for the Social Sciences Inc., Chicago, IL, EUA).

\section{RESULTADOS E DISCUSSÃO}

A composição do CEP-EEUSP, de agosto 1998 a setembro de 2004 respeitou, nos seis anos em questão, os re- 
quisitos da multidisciplinaridade, a representação dos usuários e a representação dos dois sexos. O quesito de pelo menos $50 \%$ dos membros ter experiência em pesquisa também foi contemplado pelo CEP-EEUSP, que contou, dentre os seus membros, com Professores Titulares, Professores Associados, Doutores e Mestres. Ressalta-se que a composição refere-se a membros e suplentes, que doravante serão denominados de membros.

A heterogeneidade profissional, a representação dos dois sexos e a titulação acadêmica propostas, além da representação dos usuários, objetivam maior abrangência da análise ética dos protocolos, sendo necessárias, também, as condições para o exercício da ética, que são a liberdade para discussão, a humildade para respeitar a posição do outro e a grandeza para alterar a sua, caso essa se mostre inadequada ${ }^{(9)}$, não devendo ser apresentadas posições peremptórias que impeçam a interlocução ou a confluência de posições diferentes.

Em relação à distribuição anual dos 401 projetos de pesquisa submetidos ao CEP-EEUSP no período de estudo, os quatro primeiros projetos foram registrados no Comitê em dezembro de 1998, tendo sido apreciados apenas na reunião de fevereiro do ano seguinte, uma vez que não foram apresentados em tempo hábil para serem analisados na última reunião do ano. Nesse sentido, foram incluídos entre os projetos apreciados em 1999, totalizando 41 nesse ano.

Nos anos subseqüentes, observa-se um aumento, com oscilações, no número de projetos. Assim, no ano de 2000 foram submetidos à apreciação 87 projetos; no ano de 2001 foram 84; no ano de 2002 foram 69; em 2003 foi um total de 73; e, em 2004, até 31 de agosto, data limite para entrega dos projetos a serem apreciados na reunião de setembro, deram entrada 47 projetos. Desde a constituição do Comitê, em 1998, até agosto de 2004 foram protocolados 401 projetos.

Ressalta-se que esses números não traduzem o total de projetos desenvolvidos por pesquisadores da EEUSP no período, uma vez que muitos deles submetem seus projetos aos CEPs das instituições onde pretendem coletar os dados, principalmente se essa for uma exigência da instituição campo de estudo, fazendo com que, dessa forma, nem todos os projetos sejam encaminhados para o CEP-EEUSP.

Os 401 (100\%) projetos protocolados no Comitê foram essencialmente de docentes e alunos de pós-graduação da própria Escola. Os pesquisadores externos encaminharam ao CEP-EEUSP quando tencionavam realizar a coleta de dados nessa instituição. Assim, 396 (98,7\%) projetos eram de pesquisadores da EEUSP e apenas cinco $(1,3 \%)$ de pesquisadores externos.

Em relação aos projetos efetivamente apreciados pelo CEP-EEUSP ( $n=399)$, eles puderam ter, após a primeira apreciação, uma das seguintes condições: aprovado; aprovado com pendência; com pendência; reprovado e dispensado, quando o projeto não envolvia seres humanos e, portanto, não necessitava de aprovação do Comitê. Para efeito de esclarecimento, os projetos com pendência foram divididos em com pendência e aprovados com pendência. Com pendência: quando os protocolos ficavam pendentes e o atendimento às solicitações feitas precisava retornar para ser apreciado em reunião do CEP, e aprovados com pendência: quando os protocolos ficavam pendentes, mas não precisavam retornar para reunião do Comitê para a apreciação do atendimento às solicitações, uma vez que essas eram de aspectos pontuais, ficando delegado ao relator ou à coordenação do CEP fazer essa verificação. Assim, observa-se que, dos 399 projetos apreciados, 115 (28,8\%) foram aprovados; 232 (58,2\%) foram aprovados com pendência; $41(10,3 \%)$ ficaram em pendência; cinco (1,3\%) foram reprovados; e seis $(1,5 \%)$ não se aplicavam à Resolução CNS 196/96, sendo dispensados.

Considerando que 11 projetos foram excluídos, cinco por reprovação e seis por dispensa, passaram a ser analisados 388 projetos.

Quanto ao propósito do desenvolvimento dos projetos, observa-se a seguinte distribuição dos 388 analisados: $122(31,4 \%)$ de mestrado, $113(29,1 \%)$ de iniciação científica, 63 (16,2\%) de doutorado, 28 (7,2\%) de especialização, três $(0,8 \%)$ de trabalho de conclusão de curso de graduação, dois (0,5\%) de livre docência e 57 (14,7\%) foram projetos referentes a outros propósitos.

Observa-se que o maior número de projetos apresentados tinha como finalidade a dissertação de mestrado (122) que, se comparado ao número dos que tinham como finalidade a tese de doutorado (63), correspondia a praticamente o dobro. Uma das justificativas pode ser a proporção entre mestrandos e doutorandos, uma vez que o número de alunos matriculados nos Programas de Mestrado da EEUSP também é bem maior do que o número de alunos matriculados nos Programas de Doutorado. Destaca-se o número de projetos de iniciação científica (113), evidenciando o engajamento de alunos de graduação em projetos de pesquisa e reiterando a importância da discussão sobre ética em pesquisa com os mesmos.

Em relação ao financiamento das pesquisas, a Folha de Rosto, que deve ser preenchida pelos pesquisadores para submissão dos projetos à apreciação dos CEPs, de apenas 14 (3,6\%) dos 388 (100\%) protocolos possuíam o item Patrocinador preenchido; 356 (91,8\%) indicavam não se aplica e 18 (4,6\% ) constavam em branco. Dos que preencheram o item Patrocinador, nove referiram ser a FAPESP e cinco o CNPq. Assim, foi evidenciado que os projetos apreciados, pelas próprias características, quando eram financiados, o eram por agências de fomento à pesquisa, não tendo outros financiadores que não esses, a não ser os próprios pesquisadores. Nesse item, referente ao patrocinador, parece que a finalidade precípua da informação é a identificação de possível existência de conflitos de interesses que possam existir no financiamento para o desenvolvimento dos projetos. Essa preocupação pode ser 
compreendida pelo resultado da análise do perfil dos patrocinadores dos projetos apreciados na CONEP, na qual foi constatado que a grande maioria (92\%) é financiada pela indústria farmacêutica ${ }^{(10)}$.

Quanto à área temática dos projetos, conforme orientação no verso da Folha de Rosto para preenchimento desse item, devem ser levados em conta critérios de risco, sendo considerados os de áreas temáticas especiais, aqueles pertencentes a áreas de maior prevalência de problemas éticose de menor consenso sobre condutas éticas ${ }^{(11)}$. Assim, são considerados projetos do Grupo I aqueles que se referem às áreas temáticas especiais constantes da Resolução CNS 196/96, exceto a área temática especial relativa a novos fármacos, vacinas e testes diagnósticos, que é considerada do Grupo II. Todos os outros temas, que não se enquadram nesses dois grupos de áreas temáticas especiais, são considerados do Grupo III. No CEP-EEUSP, dos 388 (100\%) projetos, $385(99,2 \%)$ eram do Grupo III e apenas três $(0,8 \%)$ eram do Grupo I, sendo dois referentes à população indígena e um encaminhado à CONEP a critério do CEP. Esses dados mostram que os projetos apreciados pelo CEP-EEUSP, na sua absoluta maioria, apresentavam menor prevalência de riscos para os sujeitos da pesquisa.

Quanto aos métodos para a realização das pesquisas, dos 388 (100\%) projetos, 170 $(43,8 \%)$ propunham o método qualitativo, $144(37,1 \%)$ o método quantitativo e 74 $(19,1 \%)$ o quanti-qualitativo, evidenciando a predominância da abordagem qualitativa nos projetos apreciados. Essa é uma realidade, provavelmente, menos comum nos Comitês de Ética em Pesquisa e, talvez, por isso, os aspectos éticos da pesquisa nessa abordagem têm recebido pouca atenção, se comparados à seara dos experimentos biomédicos com seres humanos ${ }^{(12)}$. Independente do método a ser utilizado, os princípios éticos em pesquisa devem ser resguardados no próprio desenho da investigação, considerando o objeto, objetivos, referenciais teóricos, estratégias de investigação, divulgação dos resultados e finalidades a que se propõem os investigadores ${ }^{(13)}$.

Em relação à natureza das instituições propostas para a realização das pesquisas, 228 (58,8 \%) projetos seriam desenvolvidos em instituições públicas; 40 (10,3\%) em instituições privadas ou filantrópicas; 27 (7,0\%) em instituições públicas e privadas; em $43(11,1 \%)$ a proposta era de desenvolver em outros locais, tais como comunidade de baixa renda, comunidade indígena, comunidade rural, locais públicos, domicílios de pacientes, evento, internet, dentre outros; e em 50 (12,9\%) projetos não foi possível identificar a natureza da instituição.

Observa-se que a maior parte das instituições, locais das pesquisas, corresponde a instituições públicas. A profusão dessas instituições para o desenvolvimento de pesquisas pode ser justificada pela abrangência e complexidade do sistema público de saúde e pelo fato de corres- ponderem ao maior número de campos de estágio dos alunos da Escola de Enfermagem da USP, tendo, como conseqüência, maior proximidade dos docentes com esses campos e maior compromisso de contribuir para a melhoria da assistência prestada nessas instituições.

Quanto aos sujeitos participantes, cabe esclarecer que nos projetos os sujeitos não eram mutuamente excludentes, podendo ter sido proposta mais de uma categoria de sujeito por projeto. Observa-se que, dos 388 projetos, 123 $(31,7 \%)$ propuseram realizar a pesquisa com profissionais, sendo os enfermeiros os mais focados; 73 (18,8\%) com pacientes; 62 (16,0\%) com alunos; $38(9,8 \%)$ com trabalhadores; 31 (8,0\%) com familiares; 31 (8,0\%) com docentes; 25 $(6,4 \%)$ com mulheres; 20 (5,2\%) com prontuários; 19 (4,9\%) com crianças e adolescentes; sete $(1,8 \%)$ com idosos; dois $(0,5 \%)$ com índios; e $12(3,1 \%)$ propuseram outros sujeitos, compreendendo atletas, residentes de um município, a população em geral e representantes de alguns segmentos da sociedade, dentre outros.

Quando verificada cada categoria, individualmente, observa-se que os profissionais que, em muitos casos, parecem apresentar reduzida vulnerabilidade, foram privilegiados nos projetos. Entretanto, mesmo os grupos considerados menos vulneráveis, dependendo da situação de vínculo com o pesquisador, de carência, de necessidades e de fragilidades, podem se tornar mais vulneráveis, devendo receber a proteção especial de seus direitos e de seu bem-estar.

Referente aos motivos de pendência, cabe esclarecer que puderam ser identificados vários motivos no mesmo projeto, tanto relativos ao Termo de Consentimento Livre e Esclarecido (TCLE), quanto a outras pendências, fazendo com que o número de motivos superasse o número de projetos com pendência. Observa-se que as inadequações no Termo foram as razões mais freqüentes, dentre outras identificadas. Dessa forma, dos 388 projetos, constavam pendências relativas ao TCLE em $220(56,4 \%)$ e referentes a outras causas em 170 (43,6\%). A preponderância dos motivos de pendência relativos ao TCLE também ocorre nos projetos apreciados pela CONEP e em outros $\mathrm{CEPs}^{(11,14-15)}$. Cabe ressaltar que o mesmo projeto pode, ainda, apresentar mais de um motivo em relação ao TCLE ou a outras pendências.

Os dados mostram que, em relação ao TCLE, os motivos de pendência foram relacionados a: Linguagem em 82 (21,1\%) projetos, sendo considerada, nesse item, a necessidade de adequação para torná-la mais clara para os potenciais participantes da pesquisa; Conteúdo em 58 (14,9\%) projetos, considerando a necessidade de adequação do conteúdo, incluindo a explicitação de riscos e benefícios, de gravação de entrevista, dos procedimentos da pesquisa, de retorno e de divulgação dos resultados; Forma de contato com o pesquisador em 49 (12,6\%), que foi considerada quando não constante do 
TCLE; Objetivos em 30 (7,7\%), sendo considerada a não explicitação ou a colocação incompleta dos objetivos; Direitos dos sujeitos da pesquisa em 30 (7,7\%), quando incompletos ou ausentes; Cópia do TCLE em 17 (4,4\%), quando não havia o termo ou quando a pesquisa iria ser feita com categorias diferentes de sujeitos, necessitando de termos diferentes para cada categoria; Identificação dos pesquisadores em 15 $(3,9 \%)$, quando não estavam identificados; Telefone do CEPEEUSP em sete (1,8\%) projetos, quando não estava incluído; e Assinatura do pesquisador em quatro (1,0\%), quando não constava do TCLE a previsão de assinatura do pesquisador. Alguns itens como objetivos, direitos, identificação dos pesquisadores, forma de contato com os pesquisadores e telefone do CEP também poderiam fazer parte do item referente a conteúdo, mas foi feita a opção pela colocação como itens à parte. Fica evidente que essa dificuldade de comunicação, entre o pesquisador e o sujeito de pesquisa, gera obstáculos para a obtenção do consentimento livre e esclarecido.

Em relação a outras pendências, que não o TCLE, os motivos foram relacionados a: Metodologia em 127 $(43,9 \%)$ Carta de solicitação de autorização em 59 (20,4\%) projetos, quando não havia autorização da instituição onde a coleta de dados seria realizada; Projeto 50 (17,3\%), quando havia falta de outros esclarecimentos a respeito

\section{REFERÊNCIAS}

1. Schramm FR. Aspectos cognitivos, metodológicos e morais do trabalho dos CEPs. In: Carneiro F, organizador. A moralidade dos atos científicos. Rio de Janeiro: Ministério da Saúde/FIOCRUZ; 1999. p. 53-9.

2. Declaração de Helsinque. In: Pessini L, Barchifontaine CP. Problemas atuais de Bioética. 7ạ ed. São Paulo: Centro Universitário São Camilo/Loyola; 2005. p. 485-90.

3. Conselho de Organizações Internacionais de Ciência Médicas (CIOMS). Diretrizes Éticas Internacionais para a Pesquisa Biomédica em Seres Humanos. São Paulo: Centro Universitário São Camilo/Loyola; 2004.

4. Conselho Nacional de Saúde. Resolução n.01/88. Normas de pesquisa em saúde. Bioética. 1995;3(2):137-54.

5. Conselho Nacional de Saúde. Resolução n.196, de 10 de outubro de 1996. Diretrizes e normas regulamentadoras de pesquisas envolvendo seres humanos. In: Brasil. Ministério as Saúde. Manual operacional para Comitês de Ética em Pesquisa. Brasília; 2002. p. 83-99.

6. Polit DF, Hungler BP. Fundamentos de pesquisa em enfermagem. $3^{a}$ ed. Porto Alegre: Artes Médicas; 1995. Ética e pesquisa em enfermagem; p. 291-309.

7. Freitas CBD. A legitimidade e os valores éticos expressos na Resolução CNS 196/96. In: Carneiro F, organizador. A moralidade dos atos científicos. Rio de Janeiro: Ministério da Saúde/FIOCRUZ; 1999. p. 73-7. do projeto ou inclusão de algum item não contemplado que prejudicava a apreciação; Instrumento de coleta de dados em 25 (8,7\%), quando não estavam anexados ou havia necessidade de algum esclarecimento; Cronograma em 19 (6,6\%), quando estava incompatível; Folha de rosto em $7(2,4 \%)$, quando havia a necessidade de alguma correção nessa Folha; e Currículo dos pesquisadores em 2 $(0,7 \%)$, quando não haviam sido anexados.

\section{CONSIDERAÇÕES FINAIS}

A experiência do CEP-EEUSP mostra sua progressiva consolidação. A composição atendeu aos requisitos de multidisciplinaridade, representação dos dois sexos e experiência em pesquisa. Os projetos submetidos foram, principalmente, de dissertações de mestrado e de iniciação científica; possuíam financiamento de órgãos de fomento ou dos próprios pesquisadores; propunham a utilização, preponderantemente, do método qualitativo; pertenciam, quase exclusivamente, à área temática do Grupo III; previam, na sua maioria, a utilização de instituições públicas e, grande parte, tinham profissionais como sujeitos das pesquisas. O Termo de Consentimento Livre e Esclarecido foi responsável pela maioria das pendências.

8. Hossne WS. Prefácio à edição brasileira. In: Conselho de Organizações Internacionais de Ciência Médicas (CIOMS). Diretrizes Éticas Internacionais para a Pesquisa Biomédica em Seres Humanos. São Paulo: Centro Universitário São Camilo/Loyola; 2004. p. 7-11.

9. Hossne WS. [Entrevista a Paulo Henrique de Souza]. Cad Ética Pesquisa. 2003;6(11):10-2.

10. Freitas CB, Lobo MO. Sistema CEP/CONEP. Cad Ética Pesquisa. $2001 ; 4(7): 4-13$.

11. Freitas CB, Lobo MO, Gonçalves GB. A atuação da CONEP. Cad Ética Pesquisa 2003; 6(12):5-11.

12. Sawaia BB A ética nas ciências humanas. Cad Ética Pesquisa. 2000;3(4):14-7.

13. Boemer MR. A investigação qualitativa: zelo pelo rigor e pela ética [editorial]. Rev Esc Enferm USP. 2006;40(3).

14. Pestana JOM. O CEP da UNIFESP [Depoimento]. Cad Ética Pesquisa. 2003;6(11):8-9.

15. A experiência de Manaus [Depoimento]. Cad Ética Pesquisa. 2002;5(10):8. 